$\begin{array}{ll}\text { Research Square } & \text { Preprints are preliminary reports that have not undergone peer review. } \\ \text { They should not be considered conclusive, used to inform clinical practice, } \\ \text { or referenced by the media as validated information. }\end{array}$

\title{
Health System Cost of Post-Abortion Care (PAC): A Systematic Review
}

Estro Dariatno Sihaloho ( $\nabla$ estro.sihaloho@unpad.ac.id)

Departement of Economics, Universitas Padjadjaran

Ibnu Habibie

Departement of Economics, Universitas Padjadjaran

Fariza Zahra Kamilah

Departement of Economics, Universitas Padjadjaran

Yodi Christiani

Yayasan Inisitatif Perubahan Akses Menuju Sehat (IPAS)

\section{Research Article}

Keywords: micro-costing, Post-Abortion Care (PAC), medical cost

Posted Date: September 22nd, 2021

DOI: https://doi.org/10.21203/rs.3.rs-773107/v2

License: () (1) This work is licensed under a Creative Commons Attribution 4.0 International License. Read Full License 


\section{Abstract}

Background: Despite the increasing trend of Post Abortion Care (PAC) needs and provision, the evidence related to its health system cost is lacking. The study aims to review the health system costs of Post-Abortion Care (PAC) per patient at a national level.

Methods: A systematic review of literatures related to PAC cost published in 1994 - October 2020 was performed. Electronic databases such as PubMed, Medline, The Cochrane Library, CINAHL, and PsycINFO were used to search the literature. Following the title and abstract screening, reporting quality was appraised using the Consolidated Health Economic Evaluation Reporting Standards (CHEERS) checklist. PAC costs were extrapolated into, US dollars (\$US) and international dollars (\$I), both in 2019. Content analysis was also conducted to synthesize the qualitative findings.

Results: Twelve studies met the inclusion criteria. All studies reported direct medical cost per patient in accessing PAC, but only three of them included indirect medical cost. All studies reported either average or range of cost. In terms of range, The highest direct cost of PAC with MVA (Medical Vacuum Aspiration) services can be found in Colombia, between $\$$ US50.58-212.47, while the lowest is in Malawi (\$US15.2-139.19). The highest direct cost of PAC with D\&C (Dilatation and Curettage), services was in El Salvador (\$US65.22-240.75), while the lowest is in Bangladesh (\$US15.71-103.85). Among two studies providing average indirect cost data, Uganda with \$US105.04 is the highest average indirect medical cost, while Rwanda with \$US51.44 is the lowest on the cost of indirect medical.

Conclusions: Our review shows variability in cost of PAC across countries. This study depicts a clearer picture of how costly it is for women to access PAC service, although it is still seemingly underestimated. When a study compared the use of UE method between MVA and D\&C, it is confirmed that MVA treatments tend to have lower costs and potentially reduce a significant cost. Therefore, by looking at both clinical and economic perspective, improving and strengthening the quality and accessibility of PAC with MVA is a priority.

\section{English Plain Summary}

Postabortion care (PAC) is a set of interventions designed to manage incomplete abortion and ensuing complications or related issues, such as Uterine Evacuation (UE) following an abortion. The two most common methods of UE are Manual Vacuum Aspiration (MVA) and Dilation \& Curettage (D\&C). This study reviewed the health system cost of providing those services across the country. The cost was divided into two categories: direct medical cost (i.e., labor cost \& supplies/drugs cost) and indirect medical cost (i.e., overhead cost \& capital cost). PAC costs were extrapolated into US dollars (\$US) and international dollars (\$I) in 2019.

Twelve studies were selected through database and hand-searching. Our review showed variability in providing post-abortion care across countries. It is due to data available, number of severe cases, period of data collection, and chosen costing method. The highest direct cost of PAC with MVA services can be found in Colombia, between \$50.58-212.47, while the lowest is in Malawi (\$15.2-139.19). The highest direct cost of PAC with D\&C services was in El Salvador (\$65.22-240.75), while the lowest is in Bangladesh (\$15.71-103.85). Among two studies providing average indirect cost data, Uganda with $\$ 105.04$ is the highest average indirect medical cost, while while Rwanda is the lowest with \$US51.44.

In conclusion: Our study depicts a clearer picture of how costly it is for women to access PAC service. The chosen method of UE also plays a role, as the study reconfirming MVA as a method of choice for PAC.

\section{Background}

Despite a 35\% reduction in 7-year period, the estimated maternal deaths in 2017 remain high at 295,000 worldwide [1]. While the causes are varied, the WHO predicted unsafe abortion had contributed to $13 \%$ of all maternal deaths [2, 3]. Unsafe abortion is defined as the termination of an unwanted pregnancy procedure that is performed by unqualified persons, or in an environment with minimal medical standards, or both [3, 4]. It is estimated that unsafe abortion cases reach 25.1 million each year between 2010-14, globally, where 97\% of these occurred in developing countries [5]. In these countries, an estimated five million women are admitted to hospital for treatment of complications from induced abortions annually [2]. The Guttmacher Institute projected a higher estimation that in 2019, more than 20 million women in Low-Middle Income Countries (LMICs) with unsafe abortion need post-abortion care (PAC) treatment. Still, only about $60 \%$ of them received it [6].

Unsafe abortion has detrimental consequences and generates preventable costs at multiple levels. On the societal level, many studies have confirmed that unsafe abortion leads to a high burden of morbidity and mortality [7]. Based on a public-health systems perspective, the treatment of complications due to spontaneous and induced abortion consume a disproportionate and significant amount of the maternal and gynecological hospital resources worldwide [7-10]. At the individual level, unsafe abortion can be harmful for women as it can result in severe complications and can require treatment through prolonged hospital stays [10]. Many women can also suffer from long-term morbidity as a result of these complications [3].

Although providing safe abortion services is argued as the most effective way of preventing the burden of unsafe abortion [11], it remains challenges due to highly restrictive laws, particularly in most of developing countries [3]. Furthermore, strengthening and providing high-quality PAC services seems to be more feasible action at the moment.

The cost of providing the PAC service is varied across the countries and can be complex to identify its components. Thus, there are few studies available that specifically inspect the health system cost of PAC. Furthermore, this aim of this study was to review the cost of accessing PAC per patient at a national level. We conducted a systematic review and extrapolated the cost into the international dollar for comparing purposes. 


\section{Methods \\ Search strategy}

The review was guided by The PRISMA statement [12] and the Consolidated Health Economic Evaluation Reporting Standards (CHEERS) statement [13]. A systematic review was performed using electronic databases including, PubMed, Medline, The Cochrane Library, CINAHL, and PsycINFO. All databases were searched from January 1, 1994 (the year the PAC initiative was introduced [14]) to October 10, 2020. Hand searching from reference lists in the potential selected articles also used as an alternative search method. Other inclusion criteria are title, and only publications in the English language were included, and the study should examine at least direct medical cost (i.e., costs of drugs and supplies and costs of personnel). We excluded the grey literature, such as institutional or donor reports. Studies that are not original articles and have been superseded were also excluded.

We developed the search term for health-system-cost related from Gordon and Rowell (2015) and for post-abortion care related terms from Tripney, et al. (2011) as described in Table 1. Two researchers performed the searches and conducted literature screening individually to select potential studies. When discrepancies occurred regarding the studies inclusion, both researchers discussed to reach a consensus.

Table 1

Search Terms ${ }^{\mathrm{a}}$

\begin{tabular}{|c|c|}
\hline & Search terms \\
\hline \multirow[t]{2}{*}{$\begin{array}{l}\text { Health } \\
\text { system } \\
\text { cost }^{b}\end{array}$} & 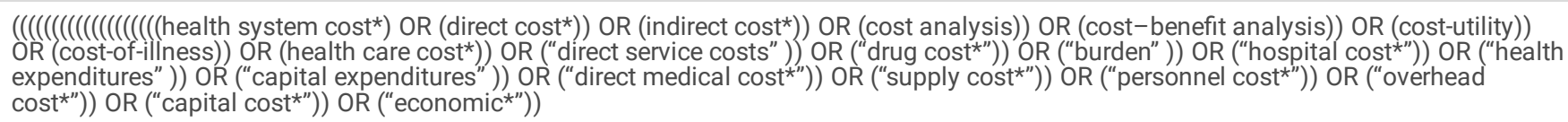 \\
\hline & AND \\
\hline $\begin{array}{l}\text { Post- } \\
\text { abortion } \\
\text { care }^{c}\end{array}$ & $\begin{array}{l}(((((((()(((((" \text { postabortion care") OR ("postabortion care"[MeSH Terms])) OR ("post abortion care"[MeSH Terms])) OR (postabortal[MeSH } \\
\text { Terms] })) \text { OR ("post abortal"[MeSH Terms]])) OR ("postabortion complication*"[MeSH Terms])) OR ("post abortion complication*"[MeSH } \\
\text { Terms] }) \text { OR ("post-abortal complication*"[MeSH Terms])) OR ("incomplete abortion*"[MeSH Terms])) OR ("incomplete abortion"[MeSH } \\
\text { Terms] })) \text { OR ("unsafe abortion*"[MeSH Terms])) OR ("unsafe abortion* complication*"[MeSH Terms]]) }\end{array}$ \\
\hline
\end{tabular}

\section{Study quality}

Research quality appraisal was guided by CHEERS statement - a specific instrument or guidelines developed for studies reporting economic outcomes as they tend to require additional economic data such as resource use, costs, and effectiveness results [13]. Hence, this instrument enabled us to critically appraised study findings, extracting components of PAC cost, and assess the eligibility for being included in extrapolated cost. However, we chose items related to our cost analysis study only. We critically appraised the studies and selected only those who were almost or fully fulfilling the criteria: reported direct medical costs, personnel costs, supplies costs, indirect medical costs, overhead costs, and capital costs.

\section{Data extraction, synthesis, and cost extrapolation}

Data extracted for each eligible study includes published year, time of data collection, cost estimation method, and estimated direct medical cost, labour or personnel cost, supplies and drugs or medications cost, indirect medical cost, overhead cost, and capital cost. We followed several studies for developing operational definitions, as shown in Table 2.

Table 2

Operational definitions of costs by category

\begin{tabular}{|c|c|c|}
\hline Name & Components & Explanations/sources \\
\hline \multirow{2}{*}{$\begin{array}{l}\text { Direct Medical } \\
\text { Cost }\end{array}$} & Labor Cost & The costs of time spent treating a patient by all medical personnel involved in a patient's care $[18,19]$ \\
\hline & $\begin{array}{l}\text { Supplies and } \\
\text { Drugs Cost }\end{array}$ & Small equipment, diagnostic tests, medications, and consumable supplies $[9,19]$ \\
\hline \multirow[t]{2}{*}{$\begin{array}{l}\text { Indirect } \\
\text { Medical Cost }\end{array}$} & Overhead Cost & $\begin{array}{l}\text { Salaries of non-medical support staff (e.g., receptionists) and administrative costs incurred by clinical staff } \\
\text { (e.g., attending meetings) }[9,18,19]\end{array}$ \\
\hline & Capital Cost & Infrastructure and equipment replacement costs and their useful lifetimes [18] \\
\hline
\end{tabular}

A deductive approach content analysis was used to summarize the data. PAC costs from each study were converted into two forms, US dollars (\$US) and international dollars (\$I), both in 2019, to improve the comparability and transferability of results across studies. Cost of PAC in US dollars (\$US), in the year of the study, was adjusted for inflation using the US gross domestic product (GDP) deflator to obtain the PAC cost in US dollars (\$US) in 2019. Afterwards, these were converted into the country's local currency unit (LCU) from each study in 2019 using official exchange rate then divided by the purchasing power parity (PPP) of each study country to obtain the PAC cost in international dollars (\$I) in 2019. All data used to convert PAC costs, such as GDP deflator, exchange rates, and PPP, are obtained from the World Development Indicator by World Bank [16]. 


\section{Results}

Based on the first search, we found 275 potentially relevant papers thorough out the sources. After reviewed based on its title as well as excluded duplication, a total of 55 distinct articles was selected for initial screening based on titles and abstracts (Fig. 1). From this step, we dropped 45 articles which mostly because either they were not a cost analysis study, or they were not specifically cover the issues of PAC or both. The other two studies were not original articles, and the other one was an institutional report.

A total of 10 full-text articles were retrieved to assess for more detailed evaluation, including examining their quality and clarity in terms of reporting using CHEERS statement. From this process, three studies were excluded because one study was based on household perspective and other chosen studies superseded two studies. We then manually searched potential articles from reference lists of the seven articles and found five chosen studies to include. Table 2 summarize information related twelve chosen studies, including their reported component cost.

Table 3

Summarize information from 12 chosen studies

\begin{tabular}{|c|c|c|c|c|c|c|c|c|c|c|c|}
\hline \multirow[t]{2}{*}{ Authors } & \multirow{2}{*}{$\begin{array}{l}\text { Published } \\
\text { year }\end{array}$} & \multirow[t]{2}{*}{ Country } & \multirow[t]{2}{*}{ Timing } & \multirow[t]{2}{*}{ Costing tools } & \multirow{2}{*}{$\begin{array}{l}\text { Base } \\
\text { Currency } \\
\text { (\$USD) }\end{array}$} & \multicolumn{6}{|c|}{ Component cost } \\
\hline & & & & & & $\begin{array}{l}\text { Direct } \\
\text { Medical } \\
\text { Cost }\end{array}$ & $\begin{array}{l}\text { Labor } \\
\text { Cost }\end{array}$ & $\begin{array}{l}\text { Supplies } \\
\text { and } \\
\text { Drugs } \\
\text { Cost }\end{array}$ & $\begin{array}{l}\text { Indirect } \\
\text { Medical } \\
\text { Cost }\end{array}$ & $\begin{array}{l}\text { Overhead } \\
\text { Cost }\end{array}$ & $\begin{array}{l}\text { Capital } \\
\text { Cost }\end{array}$ \\
\hline $\begin{array}{l}\text { Benson } \\
\text { J, et. al } \\
\text { [11] }\end{array}$ & 2015 & Malawi & $\begin{array}{l}\text { May to } \\
\text { June, } 2010\end{array}$ & $\begin{array}{l}\text { savings, } \\
\text { excel-based }\end{array}$ & 2010 & + & - & - & - & - & - \\
\hline $\begin{array}{l}\text { Ilboudo, } \\
\text { et. al [22] }\end{array}$ & 2016 & $\begin{array}{l}\text { Ouagadougou, } \\
\text { Burkina Faso }\end{array}$ & $\begin{array}{l}\text { April } 2010 \\
\text { to } 2011\end{array}$ & $\begin{array}{l}\text { Excel } \\
\text { spreadsheet }\end{array}$ & 2010 & + & + & + & - & + & - \\
\hline $\begin{array}{l}\text { Benson } \\
\text { J, et. al } \\
\text { [23] }\end{array}$ & 2012 & Nigeria & $\begin{array}{l}\text { June to } \\
\text { September, } \\
2010\end{array}$ & $\begin{array}{l}\text { savings, } \\
\text { excel-based }\end{array}$ & 2012 & + & - & - & - & - & - \\
\hline $\begin{array}{l}\text { Johnston } \\
\text { HB, et. al } \\
\text { [24] }\end{array}$ & 2012 & Bangladesh & $\begin{array}{l}\text { June to } \\
\text { August, } \\
2008\end{array}$ & $\begin{array}{l}\text { Excel } \\
\text { spreadsheet }\end{array}$ & 2008 & + & + & + & - & - & - \\
\hline $\begin{array}{l}\text { Prada E, } \\
\text { et. al [25] }\end{array}$ & 2013 & Colombia & $\begin{array}{l}\text { January to } \\
\text { April, } 2012\end{array}$ & $\begin{array}{l}\text { Post- } \\
\text { Abortion } \\
\text { Care Costing } \\
\text { Methodology } \\
\text { (PACCM) }\end{array}$ & 2012 & + & + & + & - & - & - \\
\hline $\begin{array}{l}\text { Vlassoff } \\
\text { M, et. al } \\
\text { [10] }\end{array}$ & 2012 & Ethiopia & 2008 & $\begin{array}{l}\text { Post- } \\
\text { Abortion } \\
\text { Care Costing } \\
\text { Methodology } \\
\text { (PACCM) }\end{array}$ & 2008 & + & - & - & - & - & - \\
\hline $\begin{array}{l}\text { Lince- } \\
\text { Deroche } \\
\mathrm{N}, \text { et. al } \\
\text { [17] }\end{array}$ & 2020 & Senegal & $\begin{array}{l}\text { September } \\
2016 \text { to } \\
\text { January } \\
2017\end{array}$ & $\begin{array}{l}\text { Post- } \\
\text { Abortion } \\
\text { Care Costing } \\
\text { Methodology } \\
\text { (PACCM) }\end{array}$ & 2016 & + & + & + & + & + & + \\
\hline $\begin{array}{l}\text { Baynes } \\
\text { C, et. al } \\
\text { [20] }\end{array}$ & 2019 & Tanzania & $\begin{array}{l}\text { June to } \\
\text { September, } \\
2017\end{array}$ & $\begin{array}{l}\text { Excel } \\
\text { spreadsheet }\end{array}$ & 2016 & - & + & + & - & + & + \\
\hline $\begin{array}{l}\text { Koontz } \\
\text { SL, et. al } \\
\text { [9] }\end{array}$ & 2003 & El Salvador & $\begin{array}{l}\text { February } \\
\text { to } \\
\text { November, } \\
1999\end{array}$ & $\begin{array}{l}\text { Modified } \\
\text { Ipas cost } \\
\text { model }\end{array}$ & 2003 & + & + & + & - & - & - \\
\hline $\begin{array}{l}\text { Vlassoff } \\
\text { M, et. al } \\
\text { [18] }\end{array}$ & 2015 & Rwanda & $\begin{array}{l}\text { April to } \\
\text { May, } 2012\end{array}$ & $\begin{array}{l}\text { Post- } \\
\text { Abortion } \\
\text { Care Costing } \\
\text { Methodology } \\
\text { (PACCM) }\end{array}$ & 2012 & + & + & + & + & + & + \\
\hline $\begin{array}{l}\text { Vlassoff } \\
\text { M, et. al } \\
\text { [21] }\end{array}$ & 2014 & Uganda & $\begin{array}{l}\text { August to } \\
\text { November, } \\
2010\end{array}$ & $\begin{array}{l}\text { Post- } \\
\text { Abortion } \\
\text { Care Costing } \\
\text { Methodology } \\
\text { (PACCM) }\end{array}$ & 2010 & + & + & + & + & + & + \\
\hline $\begin{array}{l}\text { Paul M, } \\
\text { et. al [7] }\end{array}$ & 2015 & Sierra Leone & $\begin{array}{l}\text { Jun to } \\
\text { July, } 2012\end{array}$ & $\begin{array}{l}\text { excel-based, } \\
\text { Modified } \\
\text { Delphi } \\
\text { approach }\end{array}$ & 2012 & + & - & - & - & - & - \\
\hline
\end{tabular}


Table 3 shows that the component cost reported were varied across the studies. The variability is caused by several factors, such as available data and chosen tools. However, all studies reported the direct medical cost of post abortion care per patient at a national level. It should be noted, regardless of not reporting the direct medical cost explicitly, we decided to include study in Tanzania [20] because they reported labour cost and supplies \& drug cost, which are components for direct medical cost based on our operational definition. Also, the variability occurred in terms of reporting the cost of each component. While some only reported their average value $[10,18,21]$, the others provided the cost by severity, resulting in range of cost when summarized in this study. Those who did not report the average values tend to avoid any misleading because the nature of PAC services is not typically generalizable, as a small number of cases can be quite disproportionate than the other cases.

\section{Field and study setting}

Twelve studies were located in Eastern Africa (Ethiopia [10]; Malawi [11]; Rwanda [18]; Uganda [21]; Tanzania [20]), Western Africa (Burkina Faso [22]; Nigeria [23]; Senegal [17]; Sierra Leone [7]), Southern Asia (Bangladesh [24]), South America (Colombia [25]), and Central America (El Salvador [9]). All of these countries are in a group of Low-Middle Income Countries (LMICs) based on World Bank classification, with seven of them are low-income countries. Based on the Guttmacher Institute, in this group, about 16\% of pregnancies ending in unsafe abortions in 2019 [6].

\section{Costing tools}

In terms of approach, costing analysis can be divided into two types, a "top-down" and a "bottom-up". When a study uses the first approach, it scrutinizes large administrative datasets and derives PAC related cost for estimating its overall cost per patient $[15,26]$. When it uses the latter approach, at first, it identifies and estimates each component then adding it up to final estimation. All chosen studies tend to use a bottom-up approach, meaning they first collect data on component costs (i.e., direct costs and indirect medical costs) then compose into the health system cost of PAC. However, some of them also used a large number of the patient database to estimate resources used, such as study in El Salvador, which was emphasized that their methodology is more specific to patient-derived costs [9].

Most of the studies used the Post-Abortion Care Costing Methodology (PACCM) as their tools. The PACCM is a bottom-up 'ingredients' approach that relies on expert opinion for estimating the health-system-cost component [21]. Study in Sierra Leone used the modified Delphi approach to solicit information [7]. Two studies in Nigeria and Malawi used savings, an excel-based tool to estimate per-case PAC costs [11, 23]. 
Table 4

Average/range cost of direct medical cost for PAC treatment per patient

\begin{tabular}{|c|c|c|c|c|c|c|c|}
\hline \multirow[t]{2}{*}{ Authors } & & \multicolumn{3}{|c|}{ (\$US in 2019) } & \multicolumn{3}{|c|}{ (\$1 in 2019) } \\
\hline & & MVA & $D \& C$ & $\begin{array}{l}\text { Method of abortion were } \\
\text { not specified }\end{array}$ & MVA & $D \& C$ & $\begin{array}{l}\text { Method of abortion were } \\
\text { not specified }\end{array}$ \\
\hline \multirow[t]{3}{*}{$\begin{array}{l}\text { Benson J, et. al } \\
\text { [11] }\end{array}$} & $\begin{array}{l}\text { Direct Medical } \\
\text { Cost }\end{array}$ & $\begin{array}{l}15.2- \\
139.19\end{array}$ & $\begin{array}{l}22.22- \\
161.42\end{array}$ & NA & $\begin{array}{l}40.75- \\
373.08\end{array}$ & $\begin{array}{l}59.56- \\
432.64\end{array}$ & NA \\
\hline & Labour Cost & NA & NA & NA & NA & NA & NA \\
\hline & $\begin{array}{l}\text { Supplies and } \\
\text { Drugs Cost }\end{array}$ & NA & NA & NA & NA & NA & NA \\
\hline \multirow[t]{3}{*}{ Ilboudo, et. al [22] } & $\begin{array}{l}\text { Direct Medical } \\
\text { Cost }\end{array}$ & $\begin{array}{l}27.16- \\
30.89\end{array}$ & NA & NA & $\begin{array}{l}79.93- \\
90.91\end{array}$ & NA & NA \\
\hline & Labour Cost & $1.23-1.6$ & NA & NA & $\begin{array}{l}3.64- \\
4.71\end{array}$ & NA & NA \\
\hline & $\begin{array}{l}\text { Supplies and } \\
\text { Drugs Cost }\end{array}$ & $\begin{array}{l}25.56- \\
29.65\end{array}$ & NA & NA & $\begin{array}{l}75.25^{-} \\
87.26\end{array}$ & NA & NA \\
\hline \multirow[t]{3}{*}{$\begin{array}{l}\text { Benson J, et. al } \\
\text { [23] }\end{array}$} & $\begin{array}{l}\text { Direct Medical } \\
\text { Cost }\end{array}$ & $\begin{array}{l}74.19- \\
137.15\end{array}$ & NA & NA & $\begin{array}{l}168.06- \\
310.66\end{array}$ & NA & NA \\
\hline & Labour Cost & NA & NA & NA & NA & NA & NA \\
\hline & $\begin{array}{l}\text { Supplies and } \\
\text { Drugs Cost }\end{array}$ & NA & NA & NA & NA & NA & NA \\
\hline \multirow[t]{3}{*}{$\begin{array}{l}\text { Johnston HB, et. } \\
\text { al [24] }\end{array}$} & $\begin{array}{l}\text { Direct Medical } \\
\text { Cost }\end{array}$ & $\begin{array}{l}15.71- \\
27.68\end{array}$ & $\begin{array}{l}15.71- \\
103.85\end{array}$ & NA & $\begin{array}{l}42.13- \\
74.23\end{array}$ & $\begin{array}{l}42.13- \\
278.46\end{array}$ & NA \\
\hline & Labour Cost & $\begin{array}{l}7.15- \\
8.48\end{array}$ & $\begin{array}{l}5.94- \\
13.46\end{array}$ & NA & $\begin{array}{l}19.18- \\
22.76\end{array}$ & $\begin{array}{l}15.95- \\
36.09\end{array}$ & NA \\
\hline & $\begin{array}{l}\text { Supplies and } \\
\text { Drugs Cost }\end{array}$ & $\begin{array}{l}7.22- \\
20.53\end{array}$ & $\begin{array}{l}13.33- \\
90.38\end{array}$ & NA & $\begin{array}{l}19.37- \\
55.05\end{array}$ & $\begin{array}{l}35.74- \\
242.33\end{array}$ & NA \\
\hline \multirow[t]{3}{*}{ Prada E, et. al [25] } & $\begin{array}{l}\text { Direct Medical } \\
\text { Cost }\end{array}$ & $\begin{array}{l}50.58- \\
212.47\end{array}$ & NA & NA & $\begin{array}{l}123.03- \\
516.75\end{array}$ & NA & NA \\
\hline & Labour Cost & NA & NA & $42.72-214.72$ & $\begin{array}{l}103.89- \\
522.21\end{array}$ & NA & NA \\
\hline & $\begin{array}{l}\text { Supplies and } \\
\text { Drugs Cost }\end{array}$ & NA & NA & $7.86-24.73$ & $\begin{array}{l}19.13- \\
60.15\end{array}$ & NA & NA \\
\hline \multirow[t]{3}{*}{$\begin{array}{l}\text { Vlassoff } M \text {, et. al } \\
\text { [10] }\end{array}$} & $\begin{array}{l}\text { Direct Medical } \\
\text { Cost }\end{array}$ & NA & NA & 43.17 & NA & NA & 120.6 \\
\hline & Labour Cost & NA & NA & NA & NA & NA & NA \\
\hline & $\begin{array}{l}\text { Supplies and } \\
\text { Drugs Cost }\end{array}$ & NA & NA & NA & NA & NA & NA \\
\hline \multirow[t]{3}{*}{$\begin{array}{l}\text { Lince-Deroche N, } \\
\text { et. al [17] }\end{array}$} & $\begin{array}{l}\text { Direct Medical } \\
\text { Cost }\end{array}$ & NA & NA & $25.03-34.07$ & NA & NA & $61.17-83.27$ \\
\hline & Labour Cost & NA & NA & $3.7-7.31$ & NA & NA & $9.05-17.86$ \\
\hline & $\begin{array}{l}\text { Supplies and } \\
\text { Drugs Cost }\end{array}$ & NA & NA & $20.85-26.76$ & NA & NA & $50.95-65.4$ \\
\hline \multirow[t]{3}{*}{$\begin{array}{l}\text { Baynes C, et. al } \\
\text { [20] }\end{array}$} & $\begin{array}{l}\text { Direct Medical } \\
\text { Cost }\end{array}$ & NA & NA & NA & NA & NA & NA \\
\hline & Labour Cost & NA & NA & $8.94-30.06$ & NA & NA & $21.97-73.83$ \\
\hline & $\begin{array}{l}\text { Supplies and } \\
\text { Drugs Cost }\end{array}$ & NA & NA & $11.89-25.22$ & NA & NA & $29.21-61.95$ \\
\hline \multirow[t]{3}{*}{$\begin{array}{l}\text { Koontz SL, et. al } \\
\text { [9] }\end{array}$} & $\begin{array}{l}\text { Direct Medical } \\
\text { Cost }\end{array}$ & $\begin{array}{l}58.82- \\
105.39\end{array}$ & $\begin{array}{l}65.22- \\
240.75\end{array}$ & NA & $\begin{array}{l}128.4- \\
230.05\end{array}$ & $\begin{array}{l}142.37- \\
525.51\end{array}$ & NA \\
\hline & Labour Cost & $\begin{array}{l}7.62- \\
21.78\end{array}$ & $\begin{array}{l}9.94- \\
30.91\end{array}$ & NA & $\begin{array}{l}16.64- \\
47.55\end{array}$ & $\begin{array}{l}21.69- \\
67.47\end{array}$ & NA \\
\hline & $\begin{array}{l}\text { Supplies and } \\
\text { Drugs Cost }\end{array}$ & $\begin{array}{l}9.39- \\
18.24\end{array}$ & $\begin{array}{l}11.71- \\
102.81\end{array}$ & NA & $\begin{array}{l}20.5- \\
39.82\end{array}$ & $\begin{array}{l}25.56- \\
224.41\end{array}$ & NA \\
\hline $\begin{array}{l}\text { Vlassoff M, et. al } \\
\text { [18] }\end{array}$ & $\begin{array}{l}\text { Direct Medical } \\
\text { Cost }\end{array}$ & NA & NA & 48.71 & NA & NA & 140.86 \\
\hline
\end{tabular}




\begin{tabular}{|c|c|c|c|c|c|c|c|}
\hline \multirow[t]{2}{*}{ Authors } & & \multicolumn{3}{|c|}{ (\$US in 2019) } & \multicolumn{3}{|c|}{ (\$1 in 2019) } \\
\hline & & MVA & $D \& C$ & $\begin{array}{l}\text { Method of abortion were } \\
\text { not specified }\end{array}$ & MVA & D\&C & $\begin{array}{l}\text { Method of abortion were } \\
\text { not specified }\end{array}$ \\
\hline & Labour Cost & NA & NA & 19.19 & NA & NA & 55.49 \\
\hline & $\begin{array}{l}\text { Supplies and } \\
\text { Drugs Cost }\end{array}$ & NA & NA & 29.52 & NA & NA & 85.37 \\
\hline \multirow[t]{3}{*}{$\begin{array}{l}\text { Vlassoff M, et. al } \\
\text { [21] }\end{array}$} & $\begin{array}{l}\text { Direct Medical } \\
\text { Cost }\end{array}$ & NA & NA & 41.34 & NA & NA & 119.86 \\
\hline & Labour Cost & NA & NA & 13.05 & NA & NA & 37.84 \\
\hline & $\begin{array}{l}\text { Supplies and } \\
\text { Drugs Cost }\end{array}$ & NA & NA & 28.28 & NA & NA & 81.98 \\
\hline \multirow[t]{3}{*}{ Paul M, et. al [7] } & $\begin{array}{l}\text { Direct Medical } \\
\text { Cost }\end{array}$ & $\begin{array}{l}68.57- \\
109.04\end{array}$ & NA & NA & $\begin{array}{l}243.27- \\
386.85\end{array}$ & NA & NA \\
\hline & Labour Cost & NA & NA & NA & NA & NA & NA \\
\hline & $\begin{array}{l}\text { Supplies and } \\
\text { Drugs Cost }\end{array}$ & NA & NA & NA & NA & NA & NA \\
\hline
\end{tabular}

Table 4 describes (i) direct medical costs, (ii) labour costs and (iii) supplies and drugs costs, in 2019 US dollars and international dollars, of post-abortion care (PAC) from 12 countries. From the table above, the costs show a lot of variabilities. There are three types of costs reported in the table, namely cost by MVA method, cost by D\&C method, and unspecified abortion method costs. In terms of range, the highest direct cost of PAC with MVA services can be found in Colombia, between $\$$ US50.58-212.47, while the lowest is in Malawi (\$US15.2-139.19). The highest direct cost of PAC with D\&C services was in El Salvador (\$US65.22-240.75), while the lowest is in Bangladesh (\$US15.71-103.85).

Table 4 also shows that only three studies reported both methods: MVA and D\&C. From the three studies above (El Salvador, Bangladesh, and Malawi), the MVA cost tends to be lower than the D\&C (dilation and curettage) cost. In El Salvador, the cost is generally lower when MVA is used, ranging from \$US58.82 to \$US105.39, while D\&C costs more, ranging from \$US65.22 to \$US240.75. In Bangladesh the cost when MVA is used extremely lower, ranging from \$US15.71 to \$US27.68, while the use of D\&C can cost up to \$103.85. Last, in Malawi, the cost for MVA use, ranging from \$US15.2 to \$US139.19, while D\&C costs between \$US22.22 and \$US161.42.

Additionally, two study reports the labor cost and supplies/drugs cost of UE using MVA \& D\&C, which are Bangladesh and El salvador. For the use of MVA, the labor cost in Bangladesh ranging from $\$$ US7.15 to $\$$ US8.48 while for the use of D\&C, from $\$ U S 5.94$ to $\$$ US13.46. For the supplies/drugs cost, the use of MVA in Bangladesh also cost less (\$US7.22-20.53) compared to D\&C (\$US13.33-90.38). Similar with Bangladesh, the use of D\&C in El Salvador generally requires more labor hour, as the use of D\&C tend to require patients stay longer in hospital, consequently it will raise the labour cost [9,24], as well as it cost more on supplies/drugs cost, as a result of higher complication rates for D\&C method thus it needs general anesthesia for pain management [24] and require more other drugs/supplies. 
Table 5

Average/range cost of indirect medical cost for PAC treatment per patient

\begin{tabular}{|c|c|c|c|c|c|c|c|}
\hline \multirow[t]{2}{*}{ Authors } & & \multicolumn{3}{|c|}{ (US\$ in 2019) } & \multicolumn{3}{|c|}{ (\$l in 2019) } \\
\hline & & MVA & $D \& C$ & $\begin{array}{l}\text { Method of abortion were not } \\
\text { specified }\end{array}$ & MVA & $D \& C$ & $\begin{array}{l}\text { Method of abortion were not } \\
\text { specified }\end{array}$ \\
\hline \multirow[t]{3}{*}{ Ilboudo, et. al [22] } & $\begin{array}{l}\text { Indirect Medical } \\
\text { Cost }\end{array}$ & NA & NA & NA & NA & NA & NA \\
\hline & Overhead Cost & NA & NA & 15.53 & NA & NA & 45.71 \\
\hline & Capital Cost & NA & NA & NA & NA & NA & NA \\
\hline \multirow[t]{3}{*}{$\begin{array}{l}\text { Lince-Deroche N, et. al } \\
\text { [17] }\end{array}$} & $\begin{array}{l}\text { Indirect Medical } \\
\text { Cost }\end{array}$ & NA & NA & $6.53-7.26$ & NA & NA & $15.97-17.76$ \\
\hline & Overhead Cost & NA & NA & $2.12-2.53$ & NA & NA & $5.18-6.19$ \\
\hline & Capital Cost & NA & NA & $0.52-0.59$ & NA & NA & $1.27-1.45$ \\
\hline \multirow[t]{3}{*}{ Baynes C, et. al [20] } & $\begin{array}{l}\text { Indirect Medical } \\
\text { Cost }\end{array}$ & NA & NA & NA & NA & NA & NA \\
\hline & Overhead Cost & NA & NA & 17.63 & NA & NA & 43.31 \\
\hline & Capital Cost & NA & NA & 14.28 & NA & NA & 35.08 \\
\hline \multirow[t]{3}{*}{ Vlassoff M, et. al [18] } & $\begin{array}{l}\text { Indirect Medical } \\
\text { Cost }\end{array}$ & NA & NA & 51.44 & NA & NA & 148.76 \\
\hline & Overhead Cost & NA & NA & 36.17 & NA & NA & 104.61 \\
\hline & Capital Cost & NA & NA & 15.25 & NA & NA & 44.11 \\
\hline \multirow[t]{3}{*}{ Vlassoff M, et. al [21] } & $\begin{array}{l}\text { Indirect Medical } \\
\text { Cost }\end{array}$ & NA & NA & 105.04 & NA & NA & 304.49 \\
\hline & Overhead Cost & NA & NA & 22.09 & NA & NA & 64.05 \\
\hline & Capital Cost & NA & NA & 82.94 & NA & NA & 240.44 \\
\hline
\end{tabular}

Unlike the direct medical cost, the studies that reported indirect cost of providing PAC, did not distinguish between the use of UE method, as shown in Table 5. Among two studies that were providing average indirect cost data, Uganda with \$US105.04 is the highest country averagely, while Rwanda with \$US51.44 is the lowest on the cost of indirect medical. The high cost in Uganda was burdened by the capital cost which accounts around $80 \%$ of the indirect medical cost, while in Rwanda that of cost only accounts to less than $30 \%$.

Table 6

Average/range cost of direct medical cost, by abortion complications*

\begin{tabular}{|c|c|c|c|c|c|c|c|c|c|c|}
\hline & \multicolumn{5}{|c|}{ Average/range of direct cost by type of complication ( $\$$ in 2019) } & \multicolumn{5}{|c|}{ Average/range of direct by type of complication (\$1 in 2019) } \\
\hline & $\begin{array}{l}\text { Incomplete } \\
\text { Abortion }\end{array}$ & Shock & Sepsis & Lacerations & Perforations & $\begin{array}{l}\text { Incomplete } \\
\text { Abortion }\end{array}$ & Shock & Sepsis & Lacerations & Perforations \\
\hline $\begin{array}{l}\text { Ilboudo, et. } \\
\text { al [22] }\end{array}$ & $\begin{array}{l}24.99- \\
30.44\end{array}$ & $\begin{array}{l}29.07- \\
52.11\end{array}$ & $\begin{array}{l}88.61- \\
110.4\end{array}$ & $\begin{array}{l}38.65- \\
69.42\end{array}$ & 86.27 & $\begin{array}{l}73.56- \\
89.6\end{array}$ & $\begin{array}{l}85.58- \\
153.36\end{array}$ & $\begin{array}{l}260.8- \\
324.93\end{array}$ & $\begin{array}{l}113.77- \\
204.31\end{array}$ & 253.91 \\
\hline $\begin{array}{l}\text { Prada E, et. } \\
\text { al [25] }\end{array}$ & $7.86-21.36$ & $\begin{array}{l}308.03- \\
410.34\end{array}$ & $\begin{array}{l}69.7- \\
98.93\end{array}$ & $26.98-60.7$ & $\begin{array}{l}103.42- \\
251.82\end{array}$ & $\begin{array}{l}19.13- \\
51.94\end{array}$ & $\begin{array}{l}749.15- \\
997.95\end{array}$ & $\begin{array}{l}169.51- \\
240.6\end{array}$ & $\begin{array}{l}65.61- \\
147.64\end{array}$ & $\begin{array}{l}251.54- \\
612.44\end{array}$ \\
\hline $\begin{array}{l}\text { Vlassoff M, } \\
\text { et. al [10] }\end{array}$ & NA & $\begin{array}{l}23.41- \\
23.65\end{array}$ & $\begin{array}{l}12.43- \\
25.57\end{array}$ & 19.79 & NA & NA & $\begin{array}{l}65.41- \\
66.08\end{array}$ & $\begin{array}{l}34.74- \\
71.44\end{array}$ & 55.29 & NA \\
\hline $\begin{array}{l}\text { Lince- } \\
\text { Deroche N, } \\
\text { et. al [17] }\end{array}$ & 15.77 & 39.53 & 25.74 & 36.33 & 38.52 & 38.53 & 96.60 & 62.91 & 88.79 & 94.13 \\
\hline $\begin{array}{l}\text { Baynes C, } \\
\text { et. al [20] }\end{array}$ & $\begin{array}{l}20.43- \\
49.17\end{array}$ & $\begin{array}{l}21.52- \\
72.9\end{array}$ & $\begin{array}{l}36.78- \\
149.84\end{array}$ & $\begin{array}{l}41.94- \\
71.32\end{array}$ & $\begin{array}{l}19.99- \\
134.29\end{array}$ & $\begin{array}{l}50.19- \\
120.77\end{array}$ & $\begin{array}{l}52.85- \\
179.05\end{array}$ & $\begin{array}{l}90.33- \\
368.01\end{array}$ & $\begin{array}{l}103- \\
175.17\end{array}$ & $\begin{array}{l}49.1- \\
329.83\end{array}$ \\
\hline $\begin{array}{l}\text { Vlassoff M, } \\
\text { et. al [18] }\end{array}$ & 103.14 & 67.22 & 31.36 & 56.43 & 175.91 & 298.27 & 194.40 & 90.70 & 163.19 & 103.14 \\
\hline $\begin{array}{l}\text { Vlassoff M, } \\
\text { et. al [21] }\end{array}$ & 48.63 & 44.48 & 52.76 & 125.61 & 133.25 & 140.99 & 128.95 & 152.95 & 364.13 & 48.63 \\
\hline
\end{tabular}

Table 6 shows the results of direct medical cost distinguished by five major abortion complications included in the WHO study on costing in its "MotherBaby Package" [20]. The total amount of direct cost from complication shows a lot of variation. 
Looking from complications' point of view, in terms of the average amount of direct cost, Rwanda has the highest direct medical cost for incomplete abortion (\$US103.14), shock (\$US67.22), and perforations (\$US175.91) compared to other countries. In Senegal, the highest average cost is a result of shock (\$US39.53), while its lowest is incomplete abortion (\$US15.77). Lastly, in Uganda, complication that cost the most, in average, is perforation (\$US133.25), and the least is shock (\$US44.48).

\section{Discussion}

Out of the twelve papers that were included in this review, we found no study examining the indirect cost of PAC, which limits our knowledge in estimating the actual health system cost of PAC. Our review provides insights into the service delivery costs of providing PAC per patient at a national level across countries, particularly developing countries. As shown, the cost is varied and depended on many factors, such as data available, number of severe cases, period of data collection and costing method.

When collecting the data, all studies sought the information from key informants as their approach generally the "bottom-up". In terms of tools, almost half of chosen studies used the PACCM, which was developed by the Guttmacher Institute and is argued as a low-cost approach yet provides robust estimation for policy analysis $[10,18]$. All studies that reported indirect medical cost were used the PACCM as their tools, indicating it as the most comprehensive tool for cost analysis of PAC service.

In addition to extrapolate the cost into the US dollar (\$US) in 2019, this review also converted all costs into international dollars (\$I) for comparing purposes. This would allow us to compare the patient's purchasing power in affording post-abortion care in their respective countries to other countries. Looking into the conversion methods with international dollars, in terms of range, the starting highest direct medical cost of PAC services is no more in Nigeria \$I 168.06 (\$US74.19), but Sierra Leone at $\$ 243.27$ (\$US68.57), while the lowest is in Malawi at $\$ 40.75$ (\$US15.2). This explicitly shows that, in relation to patient's purchasing power, the expensive case of PAC service can be found in Sierra Leone. Meanwhile, Malawi has the lowest starting point of cost of PAC. The difference is explained by some studies that suggested the use of international dollar should be interpreted carefully as converting to the international dollar could result in differences that plausibly caused by high rates of inflation in some countries relative to the USA [27].

Some of the studies, explored in more detail on WHO-recommended UE methods, MVA. Not only it is relatively cost lower than D\&C, but MVA also has been clinically proven to be as effective and safer as sharp curettage for treating incomplete abortion and inducing first-trimester abortion [28]. As previously confirmed by several studies, when comparing the cost of providing UE between MVA and D\&C in these countries, MVA treatments tend to have lower costs compared to D\&C. The use of local anesthesia for MVA instead of general anesthesia when D\&C employed is seemingly reducing the cost of MVA [24]. Also, the use of MVA potentially result in a shorter hospital stay for patients and consequently a reduce of labour cost, which in combine would significantly decrease the overall direct cost $[9,24]$.

In addition, Study in Malawi made a simulation if all women seeking first-trimester induced abortion choose MVA, which results in an estimated 20\% of cost reduction [11]. Study in El Salvador inspected the use of both methods for treating incomplete abortion, which not only confirmed that MVA could safely treat incomplete abortion, with considerable decreased hospital stay and cost as compared to sharp curettage, but also revealed that MVA has a stronger link to contraceptive services and is associated contraceptive acceptance rates [9]. Therefore, improving the quality and accessibility of PAC with MVA can reduce the overall cost of PAC and leads to alleviating the burden of unsafe abortion.

This systematic review has some limitations for consideration and discussion. First, the review only included English-language articles, which may lead to a lesser search result. Second, the differences of the operational definitions in the studies reviewed can potentially affect the findings of this review. In some studies $[18,21]$, component of labour costs were also distinct, hence it is not possible to group the costs into the same component as others. Second, the varieties in reporting the cost (range vs average). To avoid any misleading, we did not calculate the average cost when the author did not provide it on their study. Despite its limitations, this study depicts a clearer picture of how costly it is for women to access PAC service. Still, the cost is potentially underestimated as all studies included in this review did not estimate other component such as long-term healthcare costs for any complications due to unsafe abortion.

\section{Conclusions}

Our review shows variability in providing post-abortion care across countries. This study depicts a clearer picture of how costly it is for women to access PAC service, although it is still seemingly underestimated. Our findings also reconfirming MVA as a method of choice for PAC. In addition to the medical safety and women-centred approach of the method, it is also shown that MVA is less costly compared to D\&C. With a projected increasing demand of PAC globally, UE method shifting to MVA will not solely benefited women but in turn will lead to a reduced burden of cost associated with PAC across levels. Hence, UE shifting method to MVA should be initiated and promoted. Among others, it should be started with an updated policies and clinical guidelines related to it, health workers capacity buildings, and supplies at the facilities level. Government, medical professionals, and health facilities' role is critical in this effort.

\section{Abbreviations}

CHEERS : Consolidated Health Economic Evaluation Reporting Standards

D\&C : Dilatation and Curettage 


$\begin{array}{ll}\text { GDP } & \text { : Gross domestic product } \\ \text { LCU } & \text { : Local currency unit } \\ \text { MVA } & \text { : Medical Vacuum Aspiration } \\ \text { PAC } & \text { : Post Abortion Care } \\ \text { PACCM } & \text { : Post- Abortion Care Costing Methodology } \\ \text { PPP } & \text { : Purchasing power parity } \\ \text { UE } & \text { : Uterine evacuation } \\ \text { WHO } & \text { : World Health Organization }\end{array}$

\section{Declarations}

\section{Ethics approval and consent to participate}

Not applicable

\section{Consent for publication}

Not applicable

\section{Availability of data and materials}

Not applicable

\section{Competing interests}

The authors declare that they have no competing interests.

\section{Funding}

Funding for the study was obtained from Ipas Indonesia.

\section{Authors' Contributions}

EDS and IH are contributed equally to this paper as the first and the second reviewer as well as conceptualized, designed the research, analyzed the data and prepared the manuscript. FZK analyzed the data and prepared the manuscript for publication. YC conceptualized, designed the research, and prepared the manuscript. The author(s) read and approved the final manuscript.

\section{Acknowledgements}

We acknowledge Ipas Indonesia for funding the research, and the internal team of Ipas Indonesia for the support during the research. We thank Dr. Marcia Soumokil, Lea Jones and Brittany Moore for their feedbacks and inputs on the manuscript.

\section{References}

1. Sedgh G, Henshaw S, Singh S, Åhman E, Shah IH. Induced abortion: estimated rates and trends worldwide. Lancet. 2007.

2. Singh S. Hospital admissions resulting from unsafe abortion: estimates from 13 developing countries. Lancet. 2006;368:1887-92.

3. World Health Organization. WHO I Unsafe abortion: global and regional estimates of the incidence of unsafe abortion and associated mortality in 2008. Who. 2014.

4. Gebreselassie H, Fetters T, Singh S, Abdella A, Gebrehiwot Y, Tesfaye S, et al. Caring for women with abortion complications in Ethiopia: National estimates and future implications. Int Fam Plan Perspect. 2010.

5. Ganatra B, Gerdts C, Rossier C, Johnson BR, Tunçalp Ö, Assifi A, et al. Global, regional, and subregional classification of abortions by safety, 2010-14: estimates from a Bayesian hierarchical model. Lancet. 2017.

6. Elizabeth A. Sully, Biddlecom A, Darroch JE, Riley T, Ashford LS, Lince-Deroche N, et al. Adding It Up: Investing in Sexual and Reproductive Health 2019 I Guttmacher Institute. 2019. https://www.guttmacher.org/report/adding-it-up-investing-in-sexual-reproductive-health-2019. Accessed 7 Nov 2020.

7. Gebreselassie H PM. Unsafe Abortion in Sierra Leone: An Examination of Costs and Burden of Treatment on Healthcare Resources. J Womens Heal Care. 2015.

8. Johnson BR, Benson J, Bradley J, Ordoñez AR. Costs and resource utilization for the treatment of incomplete abortion in Kenya and Mexico. Soc Sci Med. 1993. 
9. Koontz SL, Molina De Perez O, Leon K, Foster-Rosales A. Treating incomplete abortion in El Salvador: Cost savings with manual vacuum aspiration. Contraception. 2003.

10. Vlassoff M, Fetters T, Kumbi S, Singh S. The health system cost of postabortion care in Ethiopia. In: International Journal of Gynecology and Obstetrics. 2012.

11. Benson J, Gebreselassie H, Mañibo MA, Raisanen K, Johnston HB, Mhango C, et al. Costs of postabortion care in public sector health facilities in Malawi: A cross-sectional survey. BMC Health Serv Res. 2015.

12. Liberati A, Altman DG, Tetzlaff J, Mulrow C, Gøtzsche PC, loannidis JPA, et al. The PRISMA statement for reporting systematic reviews and metaanalyses of studies that evaluate health care interventions: explanation and elaboration. In: Journal of clinical epidemiology. 2009.

13. Husereau D, Drummond M, Petrou S, Carswell C, Moher D, Greenberg D, et al. Consolidated Health Economic Evaluation Reporting Standards (CHEERS) statement. BMJ. 2013.

14. Tripney J, Bird KS, Kwan I, Kavanagh J. The impact of post-abortion care family planning counselling and services in low-income countries: a systematic review of the evidence (Provisional abstract). 2011.

15. Gordon LG, Rowell D. Health system costs of skin cancer and cost-effectiveness of skin cancer prevention and screening: A systematic review. European Journal of Cancer Prevention. 2015.

16. World Development Indicators | DataBank. https://databank.worldbank.org/source/world-development-indicators. Accessed 17 Nov 2020.

17. Lince-Deroche N, Sene I, Pliskin E, Owolabi OO, Bankole A. The health system costs of postabortion care in Senegal. Int Perspect Sex Reprod Health. 2020.

18. Vlassoff M, Musange SF, Kalisa IR, Ngabo F, Sayinzoga F, Singh S, et al. The health system cost of post-abortion care in Rwanda. Health Policy Plan. 2015.

19. Lince-Deroche, Sene, Pliskin, Owolabi, Bankole. The Health System Costs of Postabortion Care in Senegal. Int Perspect Sex Reprod Health. 2020;46:99.

20. Baynes C, Yegon E, Kimaro G, Lusiola G, Kahwa J. The unit and scale-up cost of postabortion care in Tanzania. Glob Heal Sci Pract. 2019.

21. Vlassoff M, Mugisha F, Sundaram A, Bankole A, Singh S, Amanya L, et al. The health system cost of post-abortion care in Uganda. Health Policy Plan. 2014.

22. Ilboudo PGC, Greco G, Sundby J, Torsvik G. Estimating the costs for the treatment of abortion complications in two public referral hospitals: a crosssectional study in Ouagadougou, Burkina Faso. BMC Health Serv Res. 2016.

23. Benson J, Okoh M, Krennhrubec K, Lazzarino MAM, Johnston HB. Public hospital costs of treatment of abortion complications in Nigeria. In: International Journal of Gynecology and Obstetrics. 2012.

24. Johnston HB, Akhter S, Oliveras E. Quality and efficiency of care for complications of unsafe abortion: A case study from Bangladesh. In: International Journal of Gynecology and Obstetrics. 2012.

25. Prada E, Maddow-Zimet I, Juarez F. The cost of postabortion care and legal abortion in Colombia. Int Perspect Sex Reprod Health. 2013.

26. Vlassoff M, Walke D, Shearer J, Newlands D, Singh S. Estimates of health care system costs of unsafe abortion in Africa and Latin America. Int Fam Plan Perspect. 2009.

27. Weissman, Eva, Sentumbwe-Mugisa, Mbonye, Anthony K, Kayaga, Emmanuel, Kihuguru SM et al. Uganda safe motherhood programme costing study. 1999.

28. Greenslade FC, Benson J, Winkler J, Henderson V, Wolf M, Leonard A. Summary of clinical and programmatic experience with manual vacuum aspiration. Adv Abort Care. 1993.

\section{Figures}




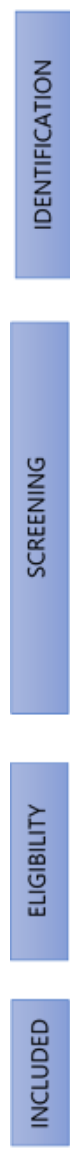

Articles identified through database searching $(n=55)$

$(n=5)$

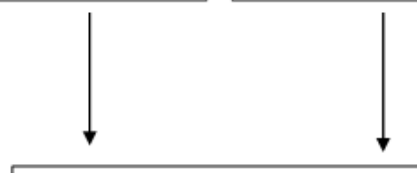

Articles included after removing

duplication

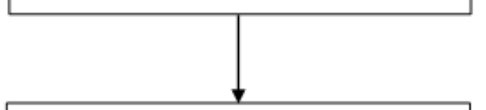

Articles included in screening for inclusion criteria based on title, abstract

and type of article

Studies excluded due to: Not cost studies and/or not PAC $(\mathrm{N}=42)$

Not original article $(\mathrm{N}=2)$

Institutional report $(\mathrm{N}=1)$

Full-text retrieved and tested for

eligibility criteria

Full-text excluded: household-

based study $(\mathrm{N}=1)$

Articles included in the review

$(n=12)$

Figure 1

PRISMA diagram

\section{Supplementary Files}

This is a list of supplementary files associated with this preprint. Click to download.

- AdditionalFile4v2.docx

- AdditionalFile2v2.docx

- AdditionalFile3v2.docx

- AdditionalFile1v2.docx 